\title{
2619. A case study of rail corrugation phenomenon based on the viewpoint of friction-induced oscillation of a wheelset-track system
}

\author{
Xiaolu Cui ${ }^{1}$, Guangxiong Chen ${ }^{2}$, Hongjuan Yang ${ }^{3}$, Huajiang Ouyang ${ }^{4}$, Wenyi Yan ${ }^{5}$ \\ ${ }^{1,2}$ Tribology Research Institute, State Key Laboratory of Traction Power, Southwest Jiaotong University, \\ Chengdu, Sichuan 610031, China \\ ${ }^{3}$ The College of Nuclear Technology and Automation Engineering, Chengdu University of Technology, \\ Chengdu, Sichuan, 610031, China \\ ${ }^{4}$ School of Engineering, University of Liverpool, The Quadrangle, Liverpool L69 3GH, UK \\ ${ }^{5}$ Department of Mechanical and Aerospace Engineering, Monash University, Clayton, VIC 3800, Australia \\ ${ }^{2}$ Corresponding author \\ E-mail: 1'cui_xiaolu@foxmail.com, ${ }^{2}$ chen_guangx@163.com, ${ }^{3} y$ hj1116@126.com, \\ ${ }^{4}$ h.ouyang@iverpool.ac.uk, ${ }^{5}$ wenyi.yan@monash.edu
}

Received 23 October 2016; received in revised form 1 April 2017; accepted 5 April 2017

DOI https://doi.org/10.21595/jve.2017.17867

\begin{abstract}
An interesting rail corrugation phenomenon unreported in open literature is found in Beijing metros, in which the wavelength of rail corrugation in the Cologne egg sleeper section is obviously different from that in the fixed dual short sleeper section, although both sleeper sections are located on an identical sharp curved track. Based on the viewpoint that friction-induced oscillation is the main cause of rail corrugation in some cases, three multiple-wheelset-track models in the transitional section on an identical sharp curved track are built in this paper. The phenomenon is researched by adopting the complex eigenvalue method together with the transient dynamic method. The simulation results are consistent with the corrugation measured in real metros. It can be found that the unstable oscillations mainly occur on the low rail and the relevant frequencies are noticeably different in different sleeper support sections. The dominant unstable oscillation frequencies in the fixed dual short sleeper and Cologne egg sleeper sections are about 201 and $422 \mathrm{~Hz}$, respectively. However, when a sharp curved track is supported only by fixed dual short sleepers or Cologne egg sleepers, the unstable oscillation frequencies are close to each other, which are both about $420 \mathrm{~Hz}$. Therefore, the corresponding wavelengths of rail corrugation are different in different conditions. The local special phenomenon can be satisfactorily explained and the viewpoint of friction-induced oscillation can be further verified.
\end{abstract}

Keywords: Cologne egg sleeper, fixed dual short sleeper, friction-induced oscillation, rail corrugation.

\section{Introduction}

\subsection{Literature review}

Rail corrugation is a critical issue in railway engineering and has been investigated by worldwide scholars for more than a century. Several formation mechanisms of corrugation have been proposed, which are very conducive to understand the generation mechanism of corrugation and to solve the problems occurring in actual conditions. According to literature reviews $[1,2]$, the generation mechanisms of rail corrugation are generally attributed to the wheel-rail transient dynamic interaction, pinned-pinned resonance, $\mathrm{P} 2$ resonance and second torsional resonance of driven axles. The opinion that the transient dynamic interaction of wheel-rail system owing to original roughness of rail surface causes corrugation is widely accepted, which assumes that the variation of friction work is caused by the transient dynamic interaction and then the corrugation appears. Numerous research works on rail corrugation were carried out based on this theory [3-11]. A linear mathematical model for descripting the wheel-rail transient dynamics was built by Muller [3], which assisted in understanding the formation of short pitch corrugation. Then, a 
time domain method for three-dimensional train-track interaction proposed by Andersson et al. [4] was used to study the mechanisms of rail corrugation related to the dynamics of a compound train-track system. Jin et al. $[5,6]$ proposed a comprehensive numerical model of train-track system to analyze the effect of corrugation on wheel-rail contact. Meanwhile, Xie et al. [7] investigated the relationship between the wear and dynamic force of a rail. And Vila et al. [8] analyzed the influence of the two coinciding resonances of the flexible rotating wheelset on corrugation growth. Meanwhile, the opinion of stick-slip oscillation causing corrugation was also widely studied to explore a potential mechanism of corrugation [12-17], which assumes that the stick-slip oscillation occurs under the full sliding conditions and then corrugation appears. Brockley [12] presented a classic equation to express the relations between the friction-induced oscillation and corrugated wear. Clark et al. [13] researched the corrugation caused by the friction-induced oscillation of flexible wheel-rail under high creepage conditions. Suda et al. [14] investigated the influence of steady creepage on the development of corrugation. Croft et al. [17] presented the creep-force relationships using a velocity-dependent friction model.

At present, some corrugation phenomena can be explained based on these existing generation mechanisms. But there are still some cannot be explained satisfactorily, such as the interesting phenomenon of rail corrugation studied in the present paper. Meanwhile, there are some distinct disadvantages in the exiting theories. The model based on the transient dynamic mechanism may generate corrugation under the excitation of a white-noise irregularity of the rail working surface. And the predicted frequency range of corrugation using the model based on the stick-slip mechanism has a certain disparity with the actual corrugation frequency range. More importantly, it appears that there is still not an effective method to prevent rails from corrugation, and hence rail grinding is applied to remove severe rail corrugations. But the cost is expensive and the corrugation may reappear after a while. Therefore, to fundamentally suppress or eliminate the corrugation, it is important to understand the mechanism and build a suitable rail corrugation model.

\subsection{Interesting rail corrugation phenomena}

According to previous researches, it is known that many factors have great influences on the formation of rail corrugation. Thereinto, the track support structure is one of important factors. In the design of railway tracks, the track support system is used to control the rail oscillation. The influences of the track support system on rail corrugation have been studied extensively [18-22]. Ilias [18] investigated the effect of sleeper vertical stiffness from $60 \mathrm{MN} / \mathrm{m}$ to $500 \mathrm{MN} / \mathrm{m}$ on the wheel-rail interaction and corrugation growth. It was concluded that the stiffer sleepers led to the larger frictional power density and higher wear rates, which sped up the corrugation growth. Enage et al. [19] also found that the softer sleepers were conducive to reduce the development of corrugation according to the academic analysis and field measurement. Vadillo et al. [20] believed that the sleeper distance had an obvious effect on the growth of rail corrugation. Jin et al. [21] pointed out that the discrete sleeper is one of the main reasons of rail corrugation growth. And the optimized parameter values of the ladder track to minimize the growth of rail corrugation were investigated by Yan et al. [22]

Because many researchers reported that a softer track support structure contributes to restraining or eliminating rail corrugation $[18,19]$, a specific track support structure characterized by a lower stiffness value, called "Cologne egg type sleeper", is used in metro tracks for oscillation isolation [23]. However, rail corrugation is severer than before. This phenomenon is inconsistent with the previous conclusion. This phenomenon and corresponding improvement have been researched by authors in previous research [24]. Based on the field observation in Beijing metro tracks, the authors recently found an unreported new phenomenon that the corrugated wavelength in the Cologne egg sleeper section is obviously different from that in the fixed dual short sleeper section, although it appears on an identical sharp curved track of radius $350 \mathrm{~m}$, as shown in Fig. 1. The dominant corrugated wavelength in the Cologne egg sleeper section is $40-50 \mathrm{~mm}$ and 
that in the fixed dual short sleeper section is $90-120 \mathrm{~mm}$. The rail corrugation on the rails supported by Cologne egg sleepers characterized by a lower supporting stiffness value is more serious than that on the rails supported by fixed dual short sleepers characterized by a higher supporting stiffness value. It was also found that if only Cologne egg sleepers or fixed dual short sleepers was used to support rails in the track of similar radius, the wavelengths of rail corrugation are very close to each other, which are both about $40-50 \mathrm{~mm}$. These rail corrugation phenomena are very puzzling. Researching on these phenomena is conducive to better understand the generation mechanism for rail corrugation and find a suitable way to suppress it.

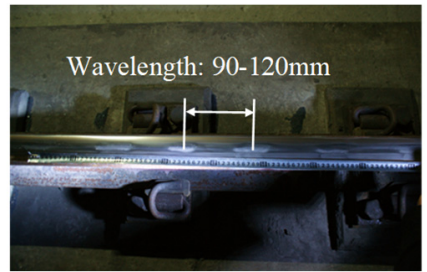

a) In the fixed dual short sleeper section

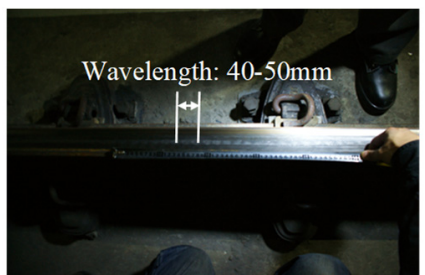

b) In the Cologne egg sleeper section

Fig. 1. Rail corrugation in different rail support sections on an identical sharp curved track

In present paper, the interesting rail corrugation phenomenon is studied based on the opinion of friction-induced oscillation leading to corrugation proposed by Chen et al. [24-27] and Kurzeck [28]. Chen et al.'s and Kurzeck's studies show that the saturated creep force may cause the friction-induced oscillation of wheelset-track system, which brings about the variation of contact forces between wheels and rails at the same frequency. Then, the friction work also varies in phase, and then corrugation occurs. Three simulation models of multiple-wheelset-track system in the transitional section from Cologne egg sleeper section to fixed dual short sleeper section on the identical sharp curved track are established. The special phenomenon of rail corrugation is investigated utilizing the complex eigenvalue analysis and transient dynamic analysis. The present work reproduces the above-mentioned interesting phenomenon of rail corrugation in theory and again proposes that the friction-induced oscillation of a wheelset-track system is a possible generation mechanism for rail corrugation.

\section{Simulation modelling of friction-induced oscillation of a multiple-wheelset-track system}

\subsection{Theoretical models of the wheelset-track system}

The creep forces on both wheels of the leading wheelsets can reach saturated when a train passes through a sharp curved track, which are equivalent to the normal contact forces times the coefficient of kinetic friction [29]. The different diagrams of leading and trailing wheelset-track systems in different sleeper sections are shown in Fig. 2. Four sub-figures clearly show the force distributions, contact conditions and rail support conditions. As for the force distributions of the wheelset-track systems, the vertical and lateral suspension forces of the left (outer) axle box are $F_{S V L}, F_{S L L}$ separately. The vertical and lateral suspension forces of the right axle (inner) box are $F_{S V R}, F_{S L R}$ separately. The normal contact forces between the wheels and rails are $N_{L}$ and $N_{R}$ separately. The creep forces between the wheels and rails are $F_{L}$ and $F_{R}$ separately. On the basis of the contact detail of leading wheelset shown in Figs. 2(a) and 2(c), it can be found that the contact point on high rail is situated between the wheel flange and profile of rail head, while that on low rail is situated between the wheel tread and rail head. And according to the contact detail of trailing wheelset shown in Figs. 2(b) and 2(d), it can be found that the contact points on the high and low rails are both situated at the wheel treads and top of rail heads. As for the track support systems, in the fixed dual short section shown in Figs. 2(a)and 2(b), the rails and sleepers are linked by a sequence of springs and dampers on contact interfaces, as well as the sleepers and ground in the fixed dual short sleeper section. $K_{R V}, K_{R L}$ and $C_{R V}, C_{R L}$ are the stiffness and damping 
values between each sleeper and a rail separately. $K_{S V}, K_{S L}$ and $C_{S V}, C_{S L}$ are the stiffness and damping values between each sleeper and the ground separately. In the Cologne egg sleeper section shown in Figs. 2(c) and 2(d), $K_{R V}, K_{R L}$ and $C_{R V}, C_{R L}$ are combined stiffness and damping values of Cologne egg sleeper separately.
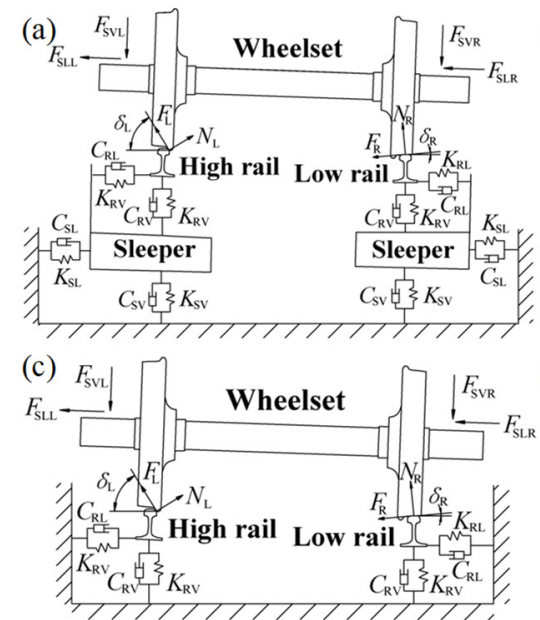

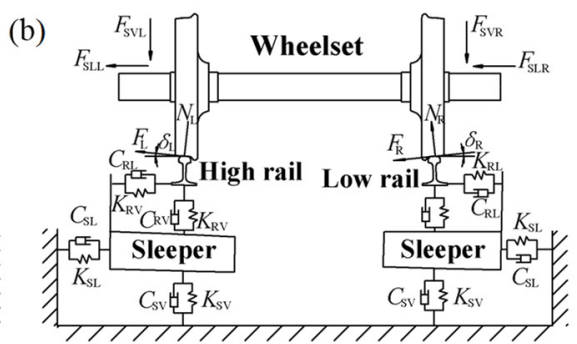

(d)

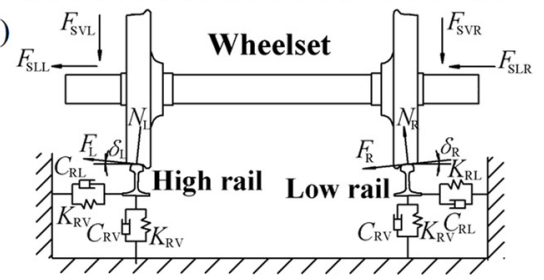

Fig. 2. Wheelset-track system on a sharp curved track: a) leading wheelset in the fixed dual short sleeper section; $b$ ) trailing wheelset in the fixed dual short sleeper section; c) leading wheelset in the Cologne egg sleeper section; d) trailing wheelset in the Cologne egg sleeper section

\subsection{Simulation model of the wheelset-track system}

According to the theoretical models shown in Fig. 2, a simulation model of multiple-wheelsettrack system in the transitional section from Cologne egg sleeper section to fixed dual short sleeper section on the identical sharp curved track of radius $350 \mathrm{~m}$ is built shown in Fig. 3, which consists of four wheelsets, two rails and a sequence of support structures.

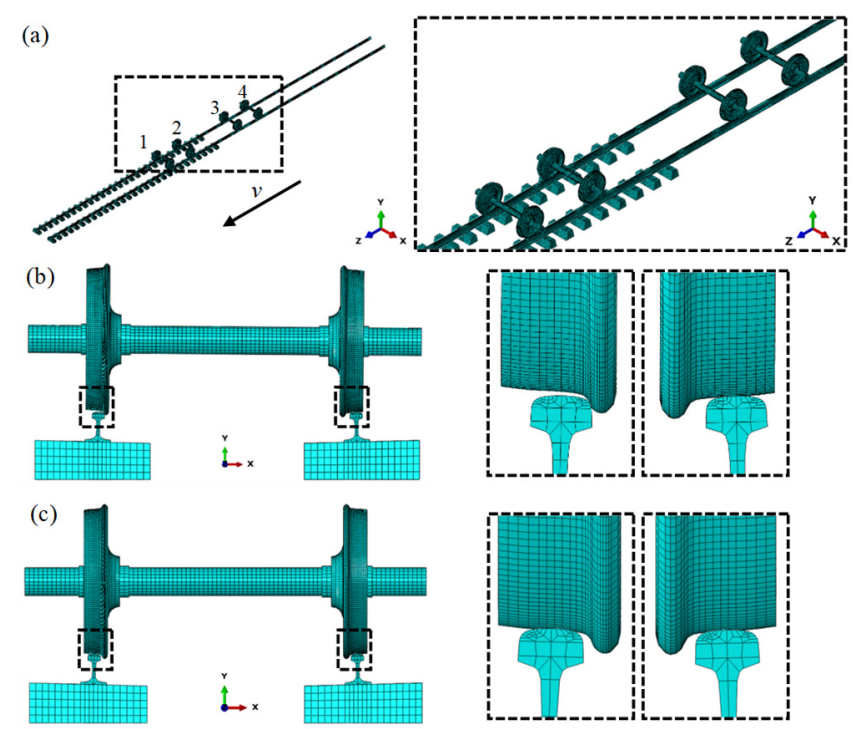

Fig. 3. Finite element model of multiple-wheelset-track system in the transitional section of a tight curved track: a) wheelsets position; b) contact details of leading wheelset in the fixed dual short section;

c) contact details of trailing wheelset in the fixed dual short section 
The position of four wheelsets in the transitional section of the sharp curved track is shown in Fig. 3(a). The first and third wheelsets are leading wheelsets, and the contact details are shown in Fig. 3(b). The second and fourth wheelsets are trailing wheelsets, and the contact details are shown in Fig. 3(c). The wheelsets of the leading bogie are situated in the track interval supported by fixed dual short sleepers. The wheelsets of the trailing bogie are situated in the track interval supported by Cologne egg sleepers. In the fixed dual short sleeper section, the sleeper is mainly made of concrete and the spacing between two sleepers is $625 \mathrm{~mm}$. The rails and fixed dual short sleepers are linked by a sequence of point to point springs and dampers on the contact interfaces. And a sequence of grounded springs and dampers are arranged on the bottom of sleeper. Meanwhile, in the Cologne egg sleeper section, the Cologne egg sleeper is mainly made of rubber and the spacing is also $625 \mathrm{~mm}$. Because the density of the rubber is less than that of the concrete and steel, the mass of a Cologne egg sleeper is much less than that of a fixed dual short sleeper. Hence in the simulation model, a Cologne egg sleeper is regarded as a group of massless spring and damper elements. The series of grounded springs and dampers are arranged at the sites where the Cologne egg sleepers are in contact with rails. The distribution details of springs and dampers between rails and sleepers, between sleepers and ground, and between rails and ground are shown in Fig. 4. Stiffness and damping values are evenly distributed to every node.

(a)

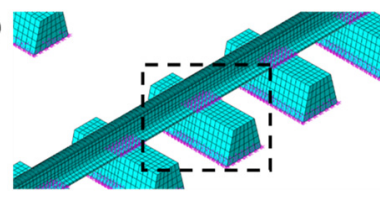

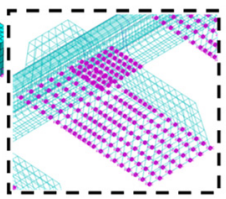

(b)

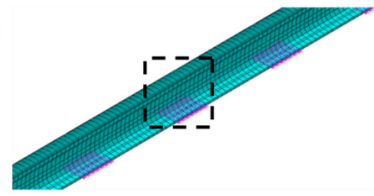

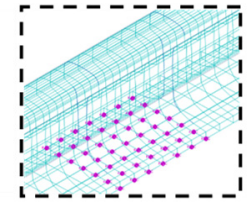

Fig. 4. Distribution details of springs and dampers: a) in the fixed dual short sleeper section,

b) in the Cologne egg sleeper section

\subsection{Nominal parameters of the wheelset-track system}

The parameters of simulation model of the wheelset-track system are obtained by actual measurement and Simpack. According to the laboratory tests and field measurements, the sub-track and material parameters of the multiple-wheelset-track system are listed in Table 1 [30]. In Simpack, the suspension forces and creep forces can be calculated. A multi-body model of the vehicle-track system on a curved track is built in Fig. 5.

Table 1. Sub-track and material parameters of the wheelset-track system

\begin{tabular}{|c|c|c|c|c|c|c|c|c|c|}
\hline \multicolumn{10}{|c|}{ Sub-track parameters in different section } \\
\hline Section & $\begin{array}{c}K_{R V} \\
(\mathrm{MN} / \mathrm{m})\end{array}$ & $\begin{array}{c}K_{R L} \\
(\mathrm{MN} / \mathrm{m})\end{array}$ & $\begin{array}{c}C_{R V} \\
(\mathrm{Ns} / \mathrm{m})\end{array}$ & \multicolumn{2}{|c|}{$\begin{array}{c}C_{R L} \\
(\mathrm{Ns} / \mathrm{m})\end{array}$} & $\begin{array}{c}K_{S V} \\
(\mathrm{MN} / \mathrm{m})\end{array}$ & $\begin{array}{c}K_{S L} \\
(\mathrm{MN} / \mathrm{m})\end{array}$ & $\begin{array}{c}C_{S V} \\
(\mathrm{Ns} / \mathrm{m})\end{array}$ & $\begin{array}{c}C_{S L} \\
(\mathrm{Ns} / \mathrm{m})\end{array}$ \\
\hline $\begin{array}{l}\text { Fixed dual } \\
\text { short sleeper }\end{array}$ & 40.73 & 8.79 & 9898.70 & \multicolumn{2}{|c|}{1927.96} & 89 & 50 & $8.98 \times 10^{4}$ & $4.0 \times 10^{4}$ \\
\hline $\begin{array}{c}\text { Cologne egg } \\
\text { sleeper }\end{array}$ & 12.07 & 7.58 & 1361.12 & \multicolumn{2}{|c|}{974.27} & - & - & - & - \\
\hline \multicolumn{10}{|c|}{ Material parameter values } \\
\hline \multicolumn{2}{|c|}{ Part } & \multicolumn{3}{|c|}{ Density $\left(\mathrm{kg} / \mathrm{m}^{3}\right)$} & & \multicolumn{2}{|c|}{ Young's modulus $(\mathrm{Pa})$} & \multicolumn{2}{|c|}{ Poisson's ratio } \\
\hline \multicolumn{2}{|c|}{ Wheelset } & \multicolumn{3}{|c|}{7800} & & \multicolumn{2}{|c|}{$2.1 \times 10^{11}$} & \multicolumn{2}{|c|}{0.3} \\
\hline \multicolumn{2}{|c|}{ Rail } & \multicolumn{3}{|c|}{7790} & & \multicolumn{2}{|c|}{$2.059 \times 10^{11}$} & \multicolumn{2}{|r|}{0.3} \\
\hline \multicolumn{2}{|c|}{ Fixed dual short sleeper } & \multicolumn{3}{|c|}{2800} & & \multicolumn{2}{|c|}{$1.9 \times 10^{11}$} & & 0.3 \\
\hline
\end{tabular}

The basic setting is same as that of finite element model built in Section 2.2. The simulation is carried out on the $350 \mathrm{~m}$ radius curved track, whose transition length is $150 \mathrm{~m}$ and the straight length is $30 \mathrm{~m}$. The nominal rail profile is CHN60. The rail gauge is $1435 \mathrm{~mm}$ and the rail can't be $1 / 40$. It is assumed that the rail is in an ideal condition (dry, smooth and rust-free). The vehicle is equipped with a car-body, suspension elements, bogies and the wheelsets. The vehicle travels 
through the curved track at a constant speed of $70 \mathrm{~km} / \mathrm{h}$. The wheel has a LM profile. It is assumed that the contact condition is single contact. The coefficient of kinetic friction between the wheels and rails is set to 0.4. In order to model track flexibility, the fixed-dual short sleeper and Cologne egg sleeper are connected to the reference system through dampers and springs, respectively. The sub-track parameters are listed in Table 1. By means of simulations, suspension forces on the track supported by different sleepers can be obtained. The average values of suspension forces are selected in the analysis, as shown in Table 2 . The vertical and lateral suspension forces are applied on every wheelset as shown in Fig. 3. And the creep forces also can be calculated. The creep forces on both wheels of the leading wheelset can reach saturated, whereas those of the training wheelset are not saturated in this case.

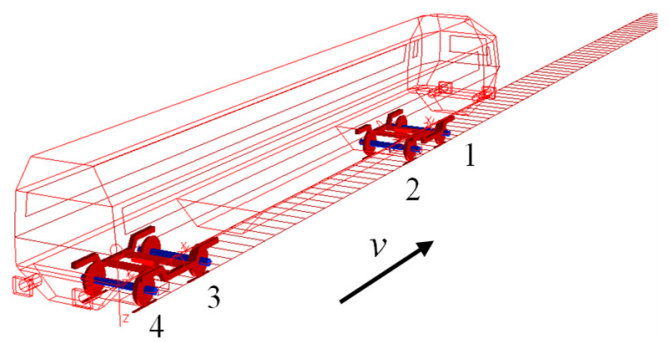

Fig. 5. Multi-body model of the vehicle-track system

Table 2. Suspension forces of each wheelset

\begin{tabular}{|c|c|c|c|c|}
\hline & $F_{S V L}(\mathrm{kN})$ & $F_{S L L}(\mathrm{kN})$ & $F_{S V R}(\mathrm{kN})$ & $F_{S L R}(\mathrm{kN})$ \\
\hline Wheelset 1 & 39.2 & 6.4 & 39.0 & 6.7 \\
\hline Wheelset 2 & 38.7 & 9.1 & 39.6 & 9.2 \\
\hline Wheelset 3 & 39.3 & 6.4 & 39.0 & 6.7 \\
\hline Wheelset 4 & 38.5 & 9.5 & 39.7 & 9.6 \\
\hline
\end{tabular}

\subsection{Finite element equations of wheelset-track system}

In the simulation analysis of friction-induced oscillation of the multiple-wheelset-track system, two different analytical methods are applied in the present paper [31]. The stability of the multiple-wheelset-track system can be forecasted adopting the complex eigenvalue analysis. From another respect, the dynamic performance of the wheelset-track system can be extracted utilizing the transient dynamic analysis.

Firstly, the theoretical methodology of the complex eigenvalue analysis method is concisely described as follows [31]. The motion equation of the multiple-wheelset-track system under the condition of sliding friction can be written as below.

$M_{f} \ddot{x}+C_{f} \dot{x}+K_{f} x=0$,

where $x$ stands for the nodal displacement vector. $M_{f}$ stands for the mass matrix; $C_{f}$ stands for the damping matrix; $K_{f}$ stands for the stiffness matrix including friction. In Eq. (1), the creep forces on the leading wheelsets of two bogies are saturated. Therefore, the system may become instable in this case.

Then, the subspace projection method is performed to calculate the complex eigenvalues. And the subspace iteration method is performed to solve the symmetric eigenvalue problem. Finally, the QZ method is applied to solve the generalized asymmetrical eigenvalue problem. Then, the general solution can be obtained as follows:

$x(t)=\sum_{i=1}^{n} y^{i}\left[\exp \left(\alpha_{i}+j \omega_{i}\right) t\right]$, 
where $y^{i}$ is the approximation of the $i$ th eigenvector of the original system. $\left(\alpha_{i}+j \omega_{i}\right)$ is the $i$ th eigenvalue. The real part $\alpha_{i}$ is the foundation to judge the stability of the system. The nodal displacement $x(t)$ will increase with time when $\alpha_{i}$ is larger than zero, which suggests that the oscillation of the system is growing to become instable. Meanwhile, the effective damping ratio $\xi_{i}$ can also be used to judge the instability of the system and defined as:

$\xi_{i}=-\alpha_{i} /\left(\pi\left|\omega_{i}\right|\right)$

The system generates the growing self-excited oscillation when $\xi_{i}$ is negative. Generally speaking, the self-excited oscillation more easily occurs when the relevant effective damping ratio is smaller.

The complex eigenvalue analysis can be used to forecast the trend of instable oscillation of linearized frictional sliding system in frequency domain using Abaqus. The C3D8I element is adopted. There are four major analysis steps. Firstly, the vertical and lateral suspension forces are applied on each wheelset. Secondly, the sliding speed is imposed on the leading wheelsets. Then, the natural frequencies and oscillation modes of the system are extracted using the mode analysis. Finally, the complex eigenvectors and instable oscillation modes are obtained through the complex eigenvalue analysis.

Then, the dynamic behaviors of frictional sliding systems in time domain can be analyzed by the transient dynamic analysis. And the method is introduced briefly [26, 31]. Firstly, the motion equation of the system can be written as below:

$M \ddot{x}_{(t)}=P_{(t)}-I_{(t)}$,

where $\ddot{x}_{(t)}$ stands for the acceleration vector, $M$ stands for the diagonal lumped mass matrix, $P_{(t)}$ stands for the external force vector, $I_{(t)}$ stands for the internal force vector. And the subscript $(t)$ stands for the time increment. Then, the motion equation is solved using the central difference method. The velocity at $(t+0.5 \Delta t)$ and displacement at $(t+\Delta t)$ of the system are calculated in the following equations:

$\dot{x}_{(t+0.5 \Delta t)}=\dot{x}_{(t-0.5 \Delta t)}+\frac{\left(\Delta t_{(t+\Delta t)}+\Delta t_{(t)}\right)}{2} \ddot{x}_{(t)}$,

$x_{(t+\Delta t)}=x_{(t)}+\Delta t_{(t+\Delta t)} \dot{x}_{(t+0.5 \Delta t)}$.

The subscripts $(t-0.5 \Delta t)$ and $(t+0.5 \Delta t)$ represent to the mid-increment values.

At present, the C3D8R element is used. The transient dynamic responses of the wheelset-track system are calculated using the explicit dynamic analysis.

\subsection{Validation of the current model}

The authors believe that the validation of a rail corrugation model should include two parts, which are the validations of a local corrugation phenomenon and the most common rail corrugation phenomena. Torstensson [11] and Vuong et al. [15] compared the predicted results of rail corrugation with the test results to validate their models. Their validation work can be considered to belong to the validation of a local corrugation phenomenon.

In metro tracks all over the world, the most common rail corrugation phenomena are presented as follows. If the curve radius of track is quite small (for example, equivalent to or less than $350 \mathrm{~m}$ in China), the probability of corrugation occurrence on low rail is quite high, but corrugation seldom appears on high rail. Furthermore, if the curve radius is larger than a certain value (for example, about 650-800 $\mathrm{m}$ in China), corrugation generates little on either rail of the tracks. However, attention was seldom paid to the validation of the most common rail corrugation phenomena in current literatures. Jin and Wen [5,6] obtained one of the best validation results of 
the general rail corrugation phenomena. They established a comprehensive model to study rail corrugation on a tight curved track, which included two wheelsets and integrated most of the existing knowledge of rail corrugation. They predicted that the low rail suffers from corrugation and the high rail is rarely subjected to corrugation on the curved track of radius $350 \mathrm{~m}$. However, they only predicted a dominant corrugation of wavelength $220-290 \mathrm{~mm}$, which is different from the corrugated wavelength of $20-80 \mathrm{~mm}$ on real metros.

According to the authors' proposed mechanism that the friction-induced oscillation of a wheelset-track system causes rail corrugation, the above-mentioned general rail corrugation phenomena can be easily validated [24-27]. Why does rail corrugation rarely occur on the rails of tangential or curved track of larger radius? The authors consider that the creep forces are not saturated in these tracks. Therefore, no friction-induced vibration occurs and no rail corrugation arises. And it is a validation of a local corrugation phenomenon in present paper, which occurs in the transitional section of a tight curved track. The authors are planning to do more validating work similar to Torstensson's work in the future.

\section{Results and discussion}

\subsection{Complex eigenvalue analysis of the motion stability of the multiple-wheelset-track system}

In this section, the tendency of the friction-induced oscillation of the multiple-wheelset-track system is studied utilizing the complex eigenvalue analysis. The instable oscillation whose basic frequency is in the range from $5 \mathrm{~Hz}$ to $1200 \mathrm{~Hz}$ is considered to be the main reason to cause corrugation. When the effective damping ratio is negative, growing self-excited oscillation occurs. Therefore, only the negative effective damping ratios are listed in the following figures. The distribution of the negative effective damping ratios in the transitional section of the sharp curved track is shown in Fig. 6. If the effective damping ratio is smaller, the relevant self-excited oscillation more easily occurs. When the frequencies are $201.73 \mathrm{~Hz}$ and $422.43 \mathrm{~Hz}$, respectively, the relevant effective damping ratios are smaller, whose values are -0.03145 and -0.04004 , respectively. Therefore, the instable oscillations more easily occur at these two frequencies in this section. The mode shape of instable oscillation is shown in Fig. 7(a) when the frequency is $201.73 \mathrm{~Hz}$. It can be found that the instable oscillation mainly occurs on the low rail. The deformation of the inner wheel of leading wheelset in the fixed dual short sleeper section is quite obvious. According to the field measurement, the vehicle passes through this section at an average speed of $70 \mathrm{~km} / \mathrm{h}$. In consequence, the corrugated wavelength is equivalent to $96.38 \mathrm{~mm}$. And when the frequency is $422.43 \mathrm{~Hz}$, the mode shape of instable oscillation is shown in Fig. 7(b). It can be found that the instable oscillation also occurs on the low rail. The deformation of inner wheel of the leading wheelset in the Cologne egg sleeper section is quite obvious too. The corresponding corrugated wavelength is equivalent to $46.03 \mathrm{~mm}$. According to the simulation results, one can find that the wavelength of the corrugation occurring on the low rail supported by the Cologne egg sleeper is quite different from that on the low rail supported by the fixed dual short sleeper in an identical sharp curved track. The conclusion is in accordance to the corrugation measured in real metros.

To further understand that the wavelengths of rail corrugation are different in different sleeper support sections of the transitional section, the self-excited oscillations of the four wheelsets in the Cologne egg sleeper section and in the fixed dual short sleeper section of the same sharp curved track are studied, respectively. The distributions of the negative effective damping ratios in different types of sleeper sections of the transitional section are shown in Fig. 8. As shown in Fig. 8(a), the smallest effective damping ratio is -0.06099 and the corresponding instable oscillation frequency is $420.96 \mathrm{~Hz}$ when the four wheelsets are all located in the Cologne egg sleeper section. The corresponding mode shape of instable oscillation is shown in Fig. 9. The instable oscillations occur on the low rail and the corresponding inner wheels of the leading 
wheelsets of both bogies. Therefore, the dominant frequency of instable oscillation is $420.96 \mathrm{~Hz}$ in the Cologne egg sleeper section.

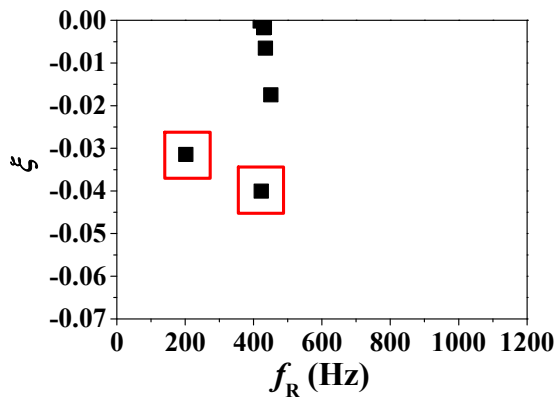

Fig. 6. Distribution of negative effective damping ratios in the transitional section

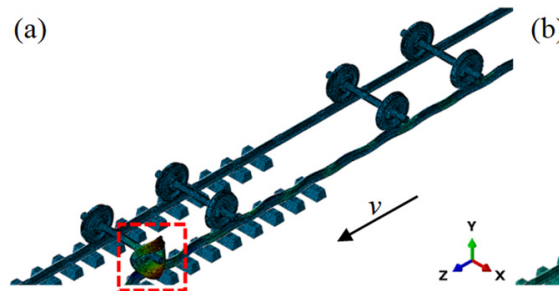

(b)

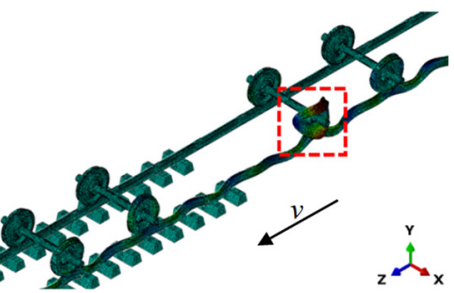

Fig. 7. Mode shapes of instable oscillations of the multiple-wheelset-track system in the transitional section: a) $f_{R}=201.73 \mathrm{~Hz}, \xi=-0.03145$; b) $f_{R}=422.43 \mathrm{~Hz}, \xi=-0.04004$
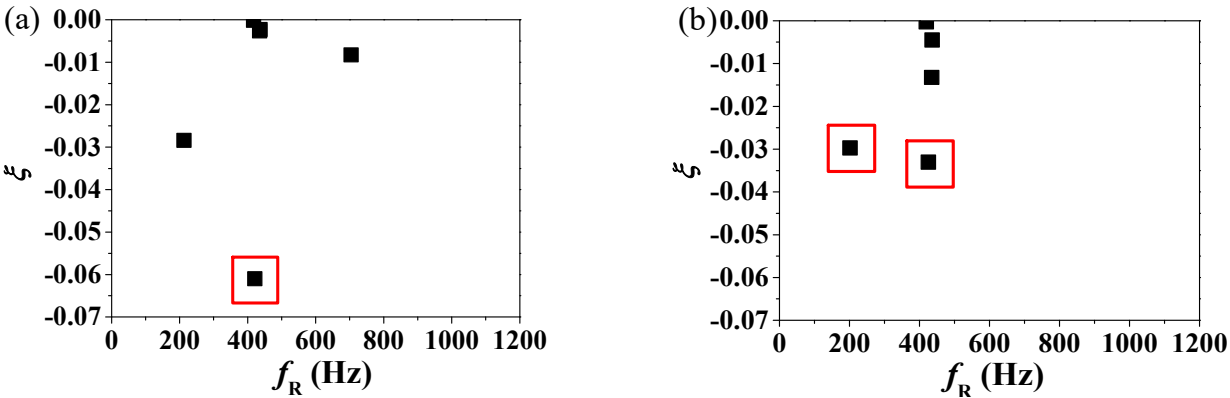

Fig. 8. Distributions of the negative effective damping ratios in different sleeper support sections of the transitional section: a) in the Cologne egg sleeper section, b) in the fixed dual short sleeper section

As shown in Fig. 8(b), the two smaller effective damping ratios are -0.02970 and -0.03320 and the corresponding instable oscillation frequencies are $201.86 \mathrm{~Hz}$ and $425.81 \mathrm{~Hz}$ when the four wheelsets are all located in the fixed dual short sleeper section. The instable oscillations may occur at these two frequencies. The mode shape of instable oscillation at a frequency of $201.86 \mathrm{~Hz}$ is shown in Fig. 10(a). The instable oscillation primarily generates on the low rail and inner wheel of the leading wheelset of the leading bogie. The mode shape of instable oscillation at a frequency of $425.81 \mathrm{~Hz}$ is shown in Fig. 10(b). The instable oscillation primarily generates on the low rail and inner wheel of the leading wheelset of the trailing bogie. When a vehicle travels from the Cologne egg sleeper section to the fixed dual short sleeper section in the transitional section, the instable oscillation in the Cologne egg sleeper section is likely to have an impact on the instable oscillation in the fixed dual short sleeper section. From Fig. 7(a), the instable oscillation at a frequency of $201.73 \mathrm{~Hz}$ first occurs in the fixed dual short sleeper section. Therefore, this instable oscillation will exist when the vehicle travels in the fixed dual short sleeper section. Why the instable oscillation at a frequency of $425.81 \mathrm{~Hz}$ cannot be developed in the fixed dual short sleeper 
section? This is due to a special phenomenon of friction-induced oscillation. The friction-induced oscillation in the fixed dual short sleeper section may be affected by the previous instable oscillation in the Cologne egg sleeper section. Hence, the dominant instable oscillation frequency is $201.86 \mathrm{~Hz}$ in the fixed dual short sleeper section. In conclusion, the instable oscillation frequencies are different in different sleeper support sections of the transitional section when a vehicle travels from the Cologne egg sleeper section to the fixed dual short sleeper section. The instable oscillation frequency is about $422 \mathrm{~Hz}$ in the Cologne egg sleeper section, while that is about $201 \mathrm{~Hz}$ in the fixed dual short sleeper section.

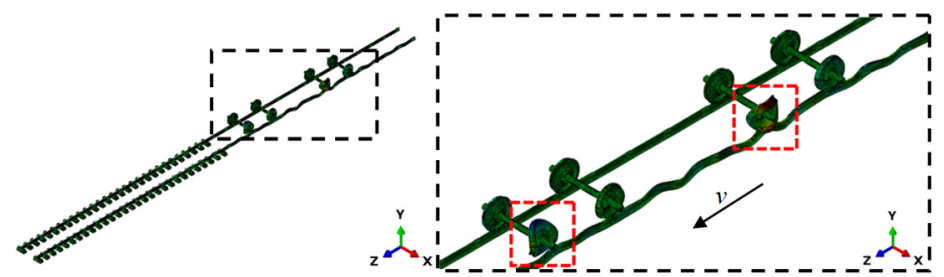

Fig. 9. Mode shape of instable oscillation of the multiple-wheelset-track system in the Cologne egg sleeper section of the transitional section: $f_{R}=420.96 \mathrm{~Hz}, \xi=-0.06099$

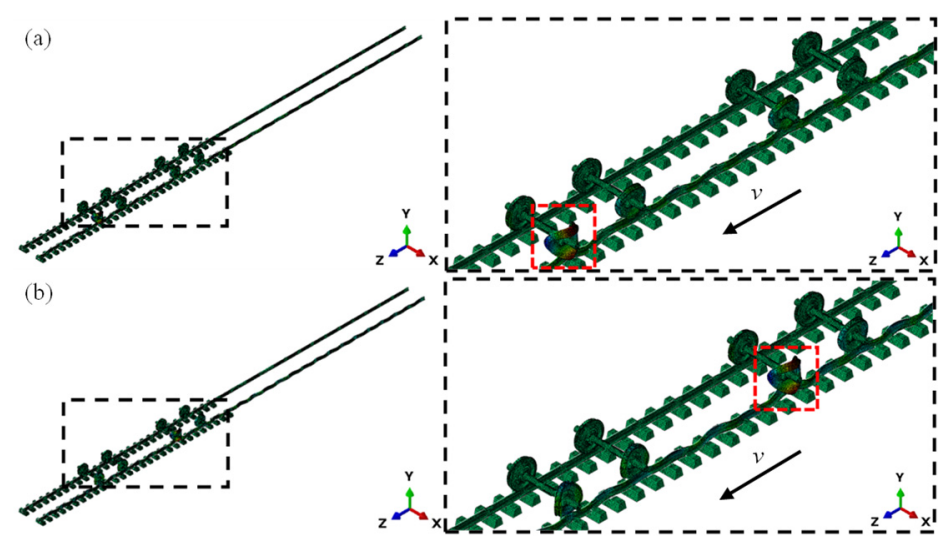

Fig. 10. Mode shapes of instable oscillation of the multiple-wheelset-track system in the fixed dual sleeper section of the transitional section: a) $f_{R}=201.86 \mathrm{~Hz}, \xi=-0.02970$; b) $f_{R}=425.81 \mathrm{~Hz}, \xi=-0.03320$

\subsection{Transient dynamic analysis of the multiple-wheelset-track system in the transitional section}

In this section, the transient dynamic analysis is adopted to research the dynamic characteristics of wheelset-track system in the transitional section when a vehicle travels from the Cologne egg sleeper section to the fixed dual short sleeper section. The running speed of the wheelset is set to $v=70 \mathrm{~km} / \mathrm{h}$ and the rotational velocity is set to $w=46.29 \mathrm{rad} / \mathrm{s}$. In this analysis, it is assumed that the track is absolutely smooth. The four wheelsets are only applied the suspension forces. In this condition, the oscillation of the wheelset-track system is a friction-induced oscillation. By means of simulation, the fluctuations of oscillation accelerations on rail surfaces in normal direction (a) can be obtained, as shown in Fig. 11. The amplitude of the oscillation acceleration on low rail is much larger than that on high rail. It further reveals that the rail corrugation usually takes place on low rail of a sharp curved track.

Then, the PSD (power spectral density) analysis is conducted to investigate the self-excited oscillation frequencies of the wheelset-track system. As shown in Fig. 12(a), there are two dominant frequencies in the oscillation acceleration of the high rail, whose values are $210.94 \mathrm{~Hz}$ and $410.16 \mathrm{~Hz}$, respectively. There are also two dominant frequencies in the oscillation acceleration of the low rail shown in Fig. 12(b), whose values are $205.08 \mathrm{~Hz}$ and $416.02 \mathrm{~Hz}$, 
respectively. The instable oscillation frequencies of the high rail are consistent with those of the low rail. Meanwhile, the friction work fluctuates at these frequencies, and then the corrugation generates $[25,26]$. The instable oscillation frequencies acquired by the transient dynamic simulation are approximate with those acquired by the complex eigenvalue simulation. It can be further demonstrated that the self-excited oscillation may lead to rail corrugation and two dominant instable oscillation frequencies exist in the transitional section of the sharp curved track. Clearly, the low rail undergoes a greater oscillation than the high rail does.

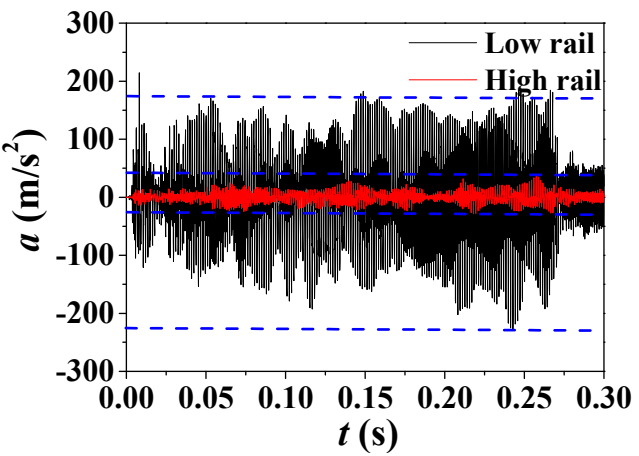

Fig. 11. Variations of the oscillation accelerations of the high and low rails with time

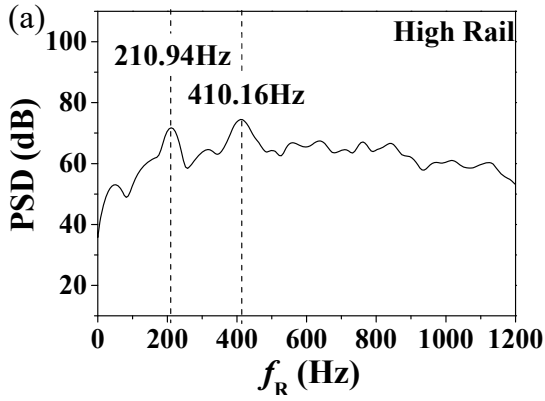

a) PSD of the instable oscillation on high rail

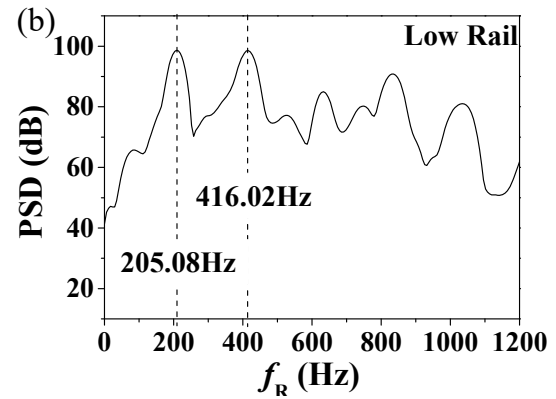

b) PSD of the instable oscillation on low rail

Fig. 12. PSD analysis results of oscillation accelerations in the transitional section

\subsection{Analysis of the multiple-wheelset-track system on a sharp curved track supported by a single type of sleepers}

In authors' field investigation into Beijing metro tracks, we found that there was an obvious difference between the wavelength of rail corrugation in the Cologne egg sleeper section and that in the fixed dual short sleeper section of an identical sharp curved track. Moreover, we also found that there is a small difference between the wavelengths of rail corrugation on the rails supported by a single type of sleepers such as single fixed dual short sleepers or single Cologne egg sleepers on a sharp curved track. We observed that dominant wavelengths of rail corrugation under these two rail support types are both about $40-50 \mathrm{~mm}$. In this section, two simulation models of the multiple-wheelset-track systems supported only by fixed dual short sleepers and supported only by Cologne egg sleepers on a sharp curved track are established, respectively, as shown in Fig. 13. The parameter values used in these simulations are same as those presented in the above sections. The rail corrugations are researched by the complex eigenvalue analysis in this section. The distribution of the negative effective damping ratios under the condition of a sharp curved track supported only by fixed dual short sleepers is shown in Fig. 14(a). The smallest effective damping ratio is -0.05521 and the corresponding instable oscillation frequency is $417.07 \mathrm{~Hz}$. From Fig. 15(a), the instable oscillation mainly takes place on the low rail. And the relevant corrugated 
wavelength is equivalent to $46.62 \mathrm{~mm}$ when the forward average velocity is $70 \mathrm{~km} / \mathrm{h}$. Meanwhile, the distribution of the effective damping ratios under the condition of a sharp curved track supported only by Cologne egg sleepers is shown in Fig. 14(b). The smallest effective damping ratio is -0.02745 and the corresponding instable oscillation frequency is $419.11 \mathrm{~Hz}$. From Fig. 15(b), the instable oscillation also mainly takes place on the low rail. The relevant corrugated wavelength is equivalent to $46.39 \mathrm{~mm}$. The present results of simulation are in accordance with real corrugations measured in Beijing metros. Therefore, the performances of corrugation occurring on the tracks individually supported by fixed dual short sleepers or Cologne egg sleepers are different from those occurring on the transitional section including the fixed dual short sleepers and Cologne egg sleepers. The arrangement of the sleepers has significant effect on rail corrugation.

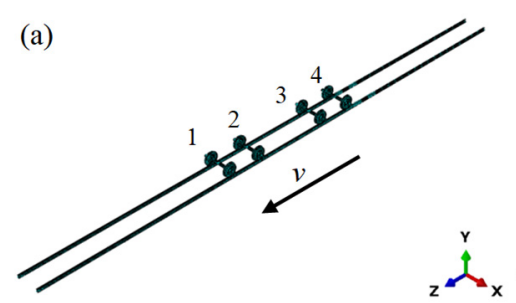

(b)

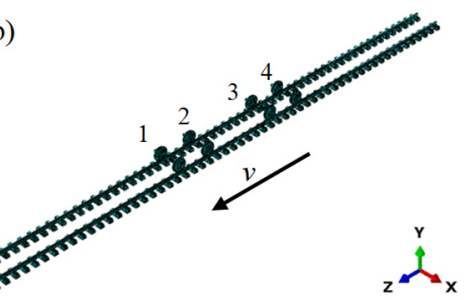

Fig. 13. Simulation model of the multiple-wheelset-track system on a sharp curved track: a) the track is only supported by Cologne egg sleepers; b) the track is only supported by fixed dual short sleepers

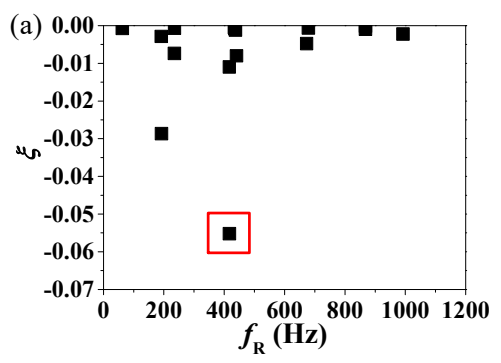

a) The track is only supported by Cologne egg sleepers

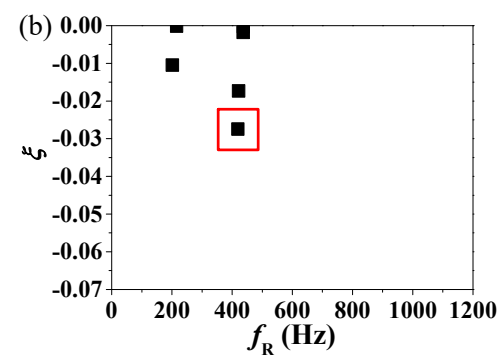

b) The track is only supported by fixed dual short sleepers

Fig. 14. Distributions of the negative effective damping ratios on a sharp curved track

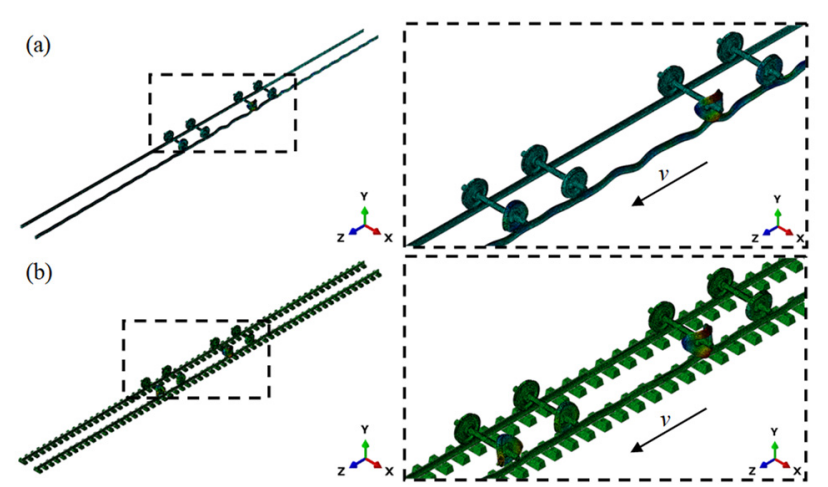

Fig. 15. Mode shapes of instable oscillation of the multiple-wheelset-track system on a sharp curved track: a) the track is supported by Cologne egg sleepers, $f_{R}=417.07 \mathrm{~Hz}, \xi=-0.05521$; b) the track is supported by fixed dual short sleepers, $f_{R}=419.11 \mathrm{~Hz}, \xi=-0.02745$

Xiaolu Cui, mainly wrote the present paper and did the numerical simulations. Guangxiong 
Chen, the corresponding author, mainly did the field observation. He also provided the key viewpoint of friction-induced vibration causing rail corrugation. Hongjuan Yang, participated in the numerical simulation and field observation. Huajiang Ouyang, provided the theoretical methodology of the complex eigenvalue analysis method. Wenyi Yan, presented some comments and suggestions and modified the grammar and spelling.

\section{Conclusions}

The present paper proposes a simulation research on the special phenomena of corrugation on the sharp curved tracks based on the opinion that friction-induced oscillation is the main cause of rail corrugation. Authors find that the present simulation results can satisfactorily explain the special phenomena of rail corrugation occurring in Beijing metro tracks and be helpful to suppress corrugation through suitable layout of track support structure in the future. Although the option needs to be further developed, it is a promising explanation of generation mechanisms of corrugation because it can satisfactorily explain some rail corrugation phenomena. In this paper, three multiple-wheelset-track simulation models are established using Abaqus, which include (1) one wheelset-track system in which two wheelsets are located on the track supported by the fixed dual short sleepers and two wheelsets are located on the track supported by the Cologne egg sleepers, (2) one wheelset-track system in which all four wheelsets are located on the track supported only by fixed dual short sleepers and (3) one wheelset-track system in which all wheelsets are located on the track supported only by Cologne egg sleepers. Two different analytical approaches are applied to investigate the rail corrugation. The conclusions are summarized up as follows:

1) When wheelsets pass through the transitional section (on a section of track in a sharp curve where the sleeper type is changed from Cologne egg sleeper to the fixed dual short sleeper), instable oscillation occurs on the low rail of the Cologne egg sleeper section at a frequency of about $422 \mathrm{~Hz}$, but occurs on the low rail of the fixed dual short sleeper section at a frequency of about $201 \mathrm{~Hz}$. It means that the dominant wavelengths of corrugation on the low rail in the Cologne egg sleeper section is $40-50 \mathrm{~mm}$ and that in the fixed dual short sleeper section is 90-120 mm.

2) When the sharp curved track is supported only by fixed dual short sleepers or only by Cologne egg sleepers, the dominant instable oscillation frequencies are both about $420 \mathrm{~Hz}$. The corrugated wavelengths are both $40-50 \mathrm{~mm}$.

3) The present simulation results confirm the theory of friction-induced oscillation causing rail corrugation. This conclusion is consistent with the actual phenomena taking place in metros. Preventing creep forces from being saturated is an essential aspect of eliminating rail corrugation.

\section{Acknowledgements}

The authors thank the financial support from National Natural Science Foundation of China (No. 51275429 and No.51505043), the 2015 Doctoral Innovation Funds of Southwest Jiaotong University and the Fundamental Research Funds for the Central Universities. The authors also thank Professor Xuesong Jin for inviting Professor Chen to take part in the field investigation into Beijing metro line 4.

\section{References}

[1] Oostermeijer K. H. Review on short pitch rail corrugation studies. Wear, Vol. 265, Issues 9-10, 2008, p. 1231-1237.

[2] Grassie S. L. Rail irregularities, corrugation and acoustic roughness: characteristics, significance and effects of reprofiling. Journal of Rail and Rapid Transit, Vol. 226, Issue 5, 2012, p. 542-557.

[3] Muller S. A linear wheel-track model to predict instability and short pitch corrugation. Journal of Sound and Vibration, Vol. 227, Issue 5, 1999, p. 899-913. 
[4] Andersson C., Johansson A. Prediction of rail corrugation generated by three-dimensional wheel-rail interaction. Wear, Vol. 257, Issues 3-4, 2004, p. 423-434.

[5] Jin X. S., Wen Z. F., Wang K. Y., Zhou Z. R., Liu Q. Y., Li C. H. Three-dimensional train-track model for study of rail corrugation. Journal of Sound and Vibration, Vol. 293, Issues 3-5, 2006, p. $830-855$.

[6] Wen Z. F., Jin X. S., Jiang Y. Elastic-plastic finite element analysis of non-steady state partial slip wheel-rail rolling contact. Journal of Tribology, Vol. 127, Issue 4, 2005, p. 713-721.

[7] Xie G., Iwnicki S. D. Calculation of wear on a corrugated rail using a three-dimensional contact model. Wear, Vol. 265, Issues 9-10, 2008, p. 1238-1248.

[8] Vila P., Baeza L. Rail corrugation growth accounting for the flexibility and rotation of the wheel set and the non-Hertzian and non-steady-state effects at contact patch. Vehicle System Dynamic, Vol. 52, Issue 1, 2014, p. 92-108.

[9] Torstensson P. T., Pieringer A., Nielsen J. C. O. Simulation of rail roughness growth on small radius curves using a non-Hertzian and non-steady wheel-rail contact model. Wear, Vol. 314, Issues 1-2, 2014, p. 241-253.

[10] Knothe K., Gross-Thebing A. Short wavelength rail corrugation and non-steady-state contact mechanics. Vehicle System Dynamic, Vol. 46, Issues 1-2, 2008, p. 49-66.

[11] Torstensson P. T., Schilike M. Rail corrugation growth on small radius curves - Measurements and validation of a numerical prediction model. Wear, Vol. 303, Issues 1-2, 2013, p. 381-396.

[12] Brockley C. A., Ko P. L. An investigation of rail corrugation using friction-induced vibration theory. Wear, Vol. 128, Issue 1, 1988, p. 99-105.

[13] Clark R., Scott G., Poole W. Short wave corrugations-an explanation based on stick-slip vibrations. Applied Mechanics Rail Transportation Symposium, 1988, p. 141-148.

[14] Suda Y., Komine H., Iwasa T., Terumichi Y. Experimental study on mechanism of rail corrugation using corrugation simulator. Wear, Vol. 253, Issues 1-2, 2002, p. 162-171.

[15] Vuong T. T., Meehan P. A., Eadie D. T., Oldknow K., Elvidge D. Investigation of a transitional wear model for wear and wear-type rail corrugation prediction. Wear, Vol. 271, Issues 1-2, 2011, p. 287-298.

[16] Saulot A., Baillet A. Dynamic finite element simulations for understanding wheel-rail contact oscillatory states occurring under sliding conditions. Journal of Tribology, Vol. 128, Issue 4, 2006, p. 761-770.

[17] Croft B., Jones C., Thompson D. J. Velocity-dependent friction in a model of wheel-rail rolling contact and wear. Vehicle System Dynamic, Vol. 49, Issue 11, 2011, p. 1791-1802.

[18] Ilias H. The influence of railpad stiffness on wheelset/track interaction and corrugation growth. Journal of Sound and Vibration, Vol. 227, Issue 5, 1999, p. 935-948.

[19] Egana J. I., Vinolas J., Seco M. Investigation of the influence of rail pad stiffness on rail corrugation on a transit system. Wear, Vol. 261, Issue 2, 2006, p. 216-224.

[20] Vadillo E. G., Tarrago J. A., Zubiaurre G. G., Duque C. A. Effect of sleeper distance on rail corrugation. Wear, Vol. 217, Issue 1, 1998, p. 140-146.

[21] Jin X. S., Wen Z. F. Effect of discrete track support by sleepers on rail corrugation at a curved track. Journal of Sound and Vibration, Vol. 315, Issues 1-2, 2008, p. 279-300.

[22] Yan Z., Markine V., Gu A., Liang Q. Optimisation of the dynamic properties of ladder track to minimise the chance of rail corrugation. Journal of Rail and Rapid Transit, Vol. 228, Issue 3, 2014, p. 285-297.

[23] Zhang H. G., Liu W. N., Liu W. F., Wu Z. Study on the cause and treatment of rail corrugation for Beijing metro. Wear, Vol. 317, Issues 1-2, 2014, p. 120-128.

[24] Cui X. L., Chen G. X., Yang H. G., Ouyang H., Zhu M. H. Study on rail corrugation of a metro tangential track with Cologne egg type fasteners. Vehicle System Dynamic, Vol. 54, Issue 3, 2016, p. 353-369.

[25] Chen G. X., Zhou Z. R., Ouyang H., Jin X. S., Zhu M. H., Zhou Z. R. A finite element study on rail corrugation based on saturated creep force-induced self-excited vibration of a wheelset-track system. Journal of Sound and Vibration, Vol. 329, Issue 22, 2010, p. 4643-4655.

[26] Qian W. J., Wu Y. F., Chen G. X., Ouyang H. Experimental and numerical studies of the effects of rail vibration absorber on suppressing short pitch rail corrugation. Journal of Vibroengineering, Vol. 18, Issue 2, 2016, p. 1133-1144. 
[27] Hu W. P., Wang P., Chen G. X., Hu Y., Cui X. L., Peng J. F., Zhu M. H. Experimental study on corrugation of a sliding surface caused by frictional self-excited vibration. Tribology Transactions, Vol. 59, Issue 1, 2015, p. 8-16.

[28] Kurzeck B. Combined friction induced oscillations of wheelset and track during the curving of metros and their influence on corrugation. Wear, Vol. 271, Issues 1-2, 2011, p. 299-310.

[29] Sun Y. Q., Simson S. Nonlinear three-dimensional wagon-track model for the investigation of rail corrugation initiation on curved track. Vehicle System Dynamic, Vol. 45, Issue 2, 2007, p. 113-132.

[30] Li W., Wang H., Wen Z., Du Wu X. L., Li X., Jin X. S. An investigation into the mechanism of metro rail corrugation using experimental and theoretical methods. Journal of Rail and Rapid Transit, Vol. 230, Issue 4, 2016, p. 1025-1039.

[31] Ouyang H. Numerical analysis of automotive disc brake squeal: a review. International Journal of Vehicle Noise and Vibration, Vol. 1, Issues 3-4, 2005, p. 207-231.

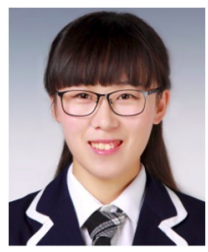

Xiaolu Cui is a Ph.D. student at School of Mechanical Engineering, Southwest Jiaotong University, Chengdu, China. Her current research interests include friction-induced vibration, wheel-rail dynamic and vibration control.

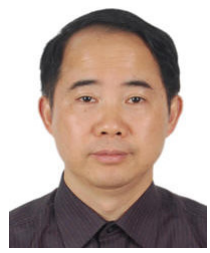

Guangxiong Chen is Professor, Ph.D. supervisor of Mechanical Engineering, Southwest Jiaotong University. His current research fields include friction vibration and noise, dynamic behavior of vehicle system.

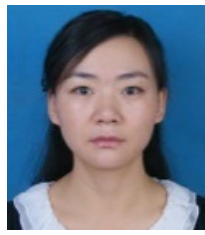

Hongjuan Yang, Ph.D., works at the College of Nuclear Technology and Automation Engineering, Chengdu University of Technology, China. Her current research interests include wear mechanism and pantograph-catenary system.

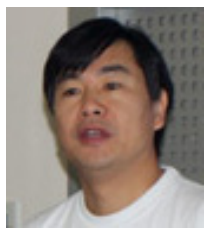

Huajiang Ouyang is Professor, Ph.D. supervisor of Mechanical Engineering, University Liverpool, UK. He acknowledges the support of Changjiang Scholarship. His current research fields include structural dynamics and control, vibration-based structural identification.

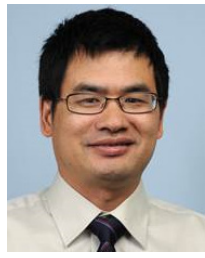

Wenyi Yan is Associate Professor, Ph.D. supervisor of Department of Mechanical and Aerospace Engineering, Monash University, Australia. His current research fields include composite structures, fracture and fatigue of materials. 